\title{
Analysis of pharynx MRI in stroke patients with obstructive sleep apnea.
}

\author{
Lixia Zhang ${ }^{1 \#}$, Qi Wu ${ }^{2 \#}$, Qian Zhung ${ }^{3}$, Gan Lu ${ }^{4}$, Yong Yuan ${ }^{5}$, Tong Wung ${ }^{*}$ \\ ${ }^{1}$ Department of Rehabilitation, The First Affiliated Hospital of Nanjing Medical University (Jiangsu Provincial \\ People's Hospital), China \\ ${ }^{2}$ Department of Rehabilitation, The First Affiliated Hospital of University of South China, China \\ ${ }^{3}$ Department of Rehabilitation, Bayinguoleng Mongol Autonomous Prefecture People's Hospital, China \\ ${ }^{4}$ Department of Respiratory Medicine, Jiangsu Provincial Authorities Hospital, China \\ ${ }^{5}$ Department of Radiology, Jiangsu Provincial Authorities Hospital, China \\ ${ }^{\#}$ These authors contributed equally.
}

\begin{abstract}
Objective: Based on the pharyngeal morphological comparison between stroke patients with OSA and without OSA, this study aimed to investigate the relation between pharyngeal anatomy and OSA in stroke patients.

Materials and Methods: Patients within 30 days of stroke underwent nocturnal polysomnography and got AHI, OAI. The magnetic resonance imaging (MRI) for clinical purposes was performed to measure retro-palatal distance, soft palatal length, soft palatal thickness, retroglossal space, tongue length, high retropharyngeal area, low retro-pharyngeal area and minimum retro-pharyngeal area. When comparing the pharyngeal measurement data in the two different groups, correlation analysis was performed among the retro-palatal distance, the retroglossal space, the tongue length and AHI, OAI, CAI etc.

Results: The retro-palatal distance of stoke patients with OSA was aberrantly shorter than the compared group $(P=0.02)$, The tongue length was longer than the compared group $(P$ value $=0.05)$. The retro-palatal distance had a negative correlation with $A H I$ and $O A I$ (respectively $R=-0.574 / P=0.00$ and $R=-0.634 / P=0.00$ ), notably, as well as the retroglossal space had a negative correlation with the AHI and CAI, but it was not related with the OAI; The linear regression showed that the relationship between AHI, OAI and the retro-palatal distance are determined a statistical significance. Conclusion: The OSA of stroke is closely associated with pharyngeal morphology change.
\end{abstract}

Keywords: Stroke, OSA, MRI, Pharyngeal morphology.

Accepted on December 25, 2017

\section{Introduction}

Obstructive sleep apnea (OSA) is an independent risk factor of stroke and is associated with poor stroke outcome. It affects an estimated $9 \%$ of women and $24 \%$ of men $[1,2]$. The prevalence of sleep-related disordered breathing (SDB, presenting with obstructive, central, or mixed apneas) in stroke patients is about $50 \%$ to $70 \%$ [3]. Increasingly, OSA is also being recognized as an independent risk factor for several clinical consequences, including systemic hypertension, cardiovascular disease, stroke, and abnormal glucose metabolism [4]. Among the patients with stroke, OSA is associated with an increased risk of mortality and the recurrence of stroke, reduced cognitive performance, and poor rehabilitation prognosis $[5,6]$. Furthermore, a large body of evidence indicated that OSA had a negative impact on the immediate and long-term functional outcome after stroke [7,8]. These important associations between OSA and stroke, and stroke outcomes underscore the need to understand the role that OSA treatment may play in stroke.
It is still unclear whether the incidence of OSA is obviously higher among the stroke patients so far. Stroke is generally not the cause of sleep apnea. Conversely, the changes in sleep physiology, neuromuscular control during sleep and increased supine sleep are likely to lead to OSA after stroke $[9,10]$. A previous study demonstrated that patients with OSA were substantially different from patients without OSA on oropharyngeal structure, including hypertrophy of the tonsil, wallowing tongue, volumes of tongue and lateral walls, hypertrophy of the uvula, decrease of the inspiratory retropalatal and retroglossal upper airway volume [11-15]. These shape difference may play a part in the mechanism of sleeprelated disordered breathing. In addition, impaired neuromuscular transmission, even to a degree insufficient to evoke respiratory symptoms, markedly impairs upper airway dimensions and function, which may also result in OSA [14]. It is noteworthy that many stroke patients are often accompanied by abnormal pharyngeal neuromuscular movement. Nevertheless, a little attention has been paid to the pharyngeal morphology of troke patients with OSA in the current literature 
Citation: Zhang L, Wu Q, Zhang Q, et al. Analysis of pharynx MRI in stroke patients with obstructive sleep apnea. Allied J Med Res. 2018;2(1):1-7.

[16]. The objective of the present study was to verify whether OSA was associated with a different oropharyngeal structure among the stroke patients. The brain MRI scans was used to compare oropharyngeal size and other anatomical characteristics in stroke patients with and without OSA. Furthermore, we could determine the correlation between the pharyngeal magnetic resonance imaging measurements which could avoid radiation exposure and the all night polysomnography measurements.

\section{Materials and Methods}

\section{Subjects}

This is a cross-sectional study. Patients with acute stroke, who hospitalized at the first affiliated hospital of Nanjing medical University were eligible as well as they had their first attack of stroke with hemiplegia confirmed by head CT or MRI. Patients were excluded decisively who had subarachnoid hemorrhage, decompensated heart failure, cardiac or respiratory arrest, or myocardial infarction within three months prior to the onset of stoke. Patients were also excluded without hesitation if they had severe pneumonia, treatment-resistant hypertension, pneumothorax, bullous emphysema, acute sinus or ear infection, or exposure to continuous positive airway pressure previously. Enrolment was examined within 30 days after the onset of stoke. Informed consent was obtained from each subject or a proxy prior to enrollment. This study was approved by the research Ethics Committee of the Nanjing Medical University.

\section{Clinical data}

Demographic and other clinical data were collected at enrolment. Stroke severity was measured by a certified physician using the National Institute of Health Stroke Scale (NIHSS) [17]. The presence of dysphagia was determined according to the Gugging Swallowing Screen [18]. Daytime sleepiness was measured using the Epworth sleepiness scale [19]. Other clinical data were collected necessarily, weight, height and neck circumference were measured by nursing staff.

\section{Polysomnography and magnetic resonance imaging assessment}

All stroke patients were studied by all night polysomnography (PSG) (Respironics Inc. USA and Alice 4 PSG recorder), and magnetic resonance imaging (MRI) (SIEMENS company 3.0T MAGNETOM MRI scanning machine) of the pharynx was performed within three days of enrollment.

PSG was used to diagnose sleep disorder for each subject during at least 7 hours sleep. The sleep apnea was measured by several indices. For example, the apnea-hypopnea index (AHI), a common measure of sleep apnea severity, which is an independent predictor of mortality in stroke patients; The obstructive apnea index (OAI); The central apnea index (CAI); The mixed apnea index (MAI) and the hypopnea index (HI). These indices which were defined as the total number of apnea hypopnea, obstructive apnea, central apnea, and mixed apnea events were divided by the total time of sleep in hours respectively. In addition, oxygen desaturation index (ODI), defined as the number of episodes per hour of sleep of oxyhemoglobin desaturation decreasing $\geq 3 \%$ from baseline of $\mathrm{SaO} 2$ and $\geq 10$ seconds in duration, was also used for assessing the sleep disorder. Lowest $\mathrm{SaO} 2$ (L-SaO2) defined as the lowest arterial oxygen saturation of all night polysomnography. Mean $\mathrm{SaO} 2$ (M-SaO2) defined as the average arterial oxygen saturation of all night polysomnography.
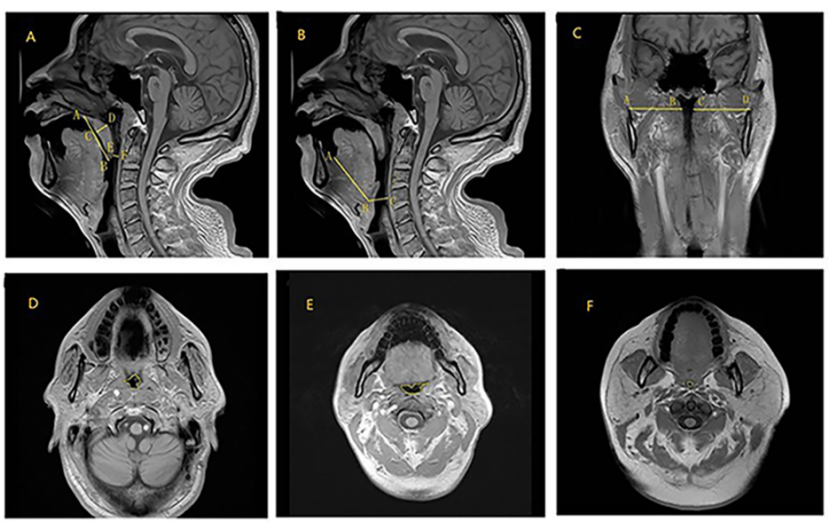

Figure 1. Pharynx MRI Measurements: (A) Length of soft palatal (AB); Soft palatal thickness (C-D) and Retro-palatal distance (E-F) on sagittal T1-weighted image; (B) Tongue length (A-B) and Retroglossal space (B-C) on sagittal T1-weighted image; (C) Lateral pharyngeal wall thickness on a coronal T1-weighted image; (D) High retro-pharyngeal area on axial T2-weighted image; (E) Low retropharyngeal area on axial T2-weighted image; (F) Minimum retropharyngeal area on axial T2-weighted image.

Interestingly, upper airway collapse is more likely to occur in the retro-palatal region in stroke patients with sleep apnea, therefore, MRI of the pharyngeal airway was performed during the acute stroke hospitalization after admitted by the patients. Images were obtained in the supine position with head in a neutral position. Both transverse and sagittal scanning were done with the following scanning parameters: cross-sectional layer thickness $5 \mathrm{~mm}$, interlayer spacing $3 \mathrm{~mm}$, scanning vision $200 \mathrm{~mm} \times 200 \mathrm{~mm}$, the sagittal layer thickness $5 \mathrm{~mm}$, layer spacing $3 \mathrm{~mm}$, and the scanning vision $60 \mathrm{~mm} \times 260$ $\mathrm{mm}$. All measurements were performed by an experienced neuro-radiologist (Initial of the neuro-radiologist if he/she is a co-author). The following MRI measurements (Figure 1) were detected with details available in the previous literature $[16,20,21]$. Images were obtained in the supine position with the head in a neutral position. Instructions for breath holding during MRI image acquisition were provided to each subject; however, no information on compliance with these directions is available. Measurements as following:

I. Retro-palatal distance, defined as the shortest vertical distance from the soft palate to the pharynx posterior wall (Figure 1A, distance between $\mathrm{E}$ and F); II. Length of soft palatal, defined as the distance from the posterior limits of the hard palate to its tip (Figure 1A, distance between A and B); III. Soft palatal thickness, measured as the perpendicular to the length of the soft palate (Figure 1A, C-D line); IV. Retroglossal space, measured as the perpendicular distance between the anterior floor of the vallecula (postero-inferior base of tongue) 
and the posterior wall of the hypopharynx (Figure 1B, A-B line); V. Tongue length (valleculartongue base distance), measured as the shortest distance between the anterior floor of the vallecula (posterior-inferior base of tongue) and the anterior-inferior corner of the tongue base (Figure 1B, B-C line); VI. Lateral pharyngeal wall thickness, defined as the distance between intercondylar notch of the mandible (A, D) and the lateral edge of the oropharyngeal airway. The total pharyngeal soft tissue thickness was defined as the sum of the thickness on both right and left side (Figure 1C, A-B line, and B-C line); VII. High retropharyngeal area, defined as $0.5 \mathrm{~cm}$ below the plane of the hard palate airway cross-sectional area (Figure 1D); VIII. Low retropharyngeal area, $1 \mathrm{~cm}$ at the top of the free edge of the epiglottis airway cross-sectional area (Figure 1E); IX. Minimum retropharyngeal area, defined as the narrowest axial cross-sectional area of pharynx at the level between the hard palate plane and epiglottis tip (Figure 1F).

\section{Statistical analysis}

Analyses were conducted using IBM SPSS Statistics 19.0 (SPSS Inc., Chicago, IL, USA). Clinical characteristics of the patients were summarized as mean and standard deviation (SD) for normally-distributed continuous data, median and interquartile ranges (IQR) for non-normally distributed continuous data, frequency and percentage for categorical data. Two-sample t-test, Wilcoxon rank sum test, or Chi-squared test was used to compare the differences between patients with OSA and patients without OSA as appropriate. Pearson correlation coefficient was used to assess the correlation between any pair of the MRI and PSG measurements. Linear regression was used to determine the relationship between the retro-palatal distance and the OAI and AHI.

\section{Results}

Twenty-three subjects met the inclusion criteria and were included in the analysis. The median time between the stroke onset and the student enrolment was 23 days (interquartile range $(\mathrm{IQR})=10,30)$. The median age was 53 years $(\mathrm{IQR}=35$, 79). Fourteen $(61 \%)$ of the subjects had OSA. For group of subjects with OSA, the median AHI was $49.5(\mathrm{IQR}=22,71)$ and the median minimum oxygen saturation was $74 \%$ $(\mathrm{IQR}=50 \%, 82 \%)$. In contrast, for the group of subjects without OSA, the median AHI was $2.1(\mathrm{IQR}=1,4)$ and the median minimum oxygen saturation was $89 \%(\mathrm{IQR}=60,91)$. Patients with and without OSA did not differ in demographics, vascular risk factors, or stroke severity. Comparison of the characteristics of patients with and without OSA was presented in Table 1.

Table1. Comparison of Baseline characteristics of patients with OSA and without OSA.

\begin{tabular}{|c|c|c|c|}
\hline Baseline Characteristics & $\begin{array}{l}\text { Patients with } \\
\text { OSA }(n=14)\end{array}$ & $\begin{array}{l}\text { Patients without } \\
\text { OSA }(n=9)\end{array}$ & $p$-value \\
\hline Female, n (\%) & $4(28.6 \%)$ & $4(44.4 \%)$ & 0.29 \\
\hline Ischemic stroke, n (\%) & $6(42.9 \%)$ & $6(66.7 \%)$ & 0.46 \\
\hline Age (years), mean (SD) & $57.2(12.6)$ & $51.8(5.0)$ & 0.17 \\
\hline $\begin{array}{l}\text { Time since stroke onset, } \\
\text { (days), mean (SD) }\end{array}$ & $24.2(5.4)$ & $22.3(7.7)$ & 0.38 \\
\hline $\begin{array}{l}\text { Neck circumference }(\mathrm{cm}) \text {, } \\
\text { mean (SD) }\end{array}$ & $39.9 \pm 5.1$ & $36.4 \pm 2.5$ & 0.48 \\
\hline Hypertension, n (\%) & $12(\%)$ & $5(\%)$ & 0.12 \\
\hline Coronary heart disease, n (\%) & $7(\%)$ & $5(\%)$ & 0.81 \\
\hline Diabetes, n (\%) & $7(\%)$ & $2(\%)$ & 0.2 \\
\hline Hyperlipidemia, n (\%) & $7(\%)$ & $1(\%)$ & 0.06 \\
\hline Drinking, n (\%) & $6(\%)$ & $1(\%)$ & 0.12 \\
\hline Smoking, n (\%) & $6(\%)$ & $3(\%)$ & 0.67 \\
\hline NIH stroke scale, mean (SD) & $14.6(4.7)$ & $10.6(5.2)$ & 0.07 \\
\hline Dysphagia, n (\%) & $1(9 \%)$ & $6(66.7 \%)$ & 0.12 \\
\hline Body mass index, mean (SD) & $25.8(1.0)$ & $23.7(3.6)$ & 0.18 \\
\hline
\end{tabular}

The retro-palatal distance for patients with OSA was significantly longer than that for patients without OSA (mean \pm $\mathrm{SD}=5.7 \pm 1.4$ versus $4.3 \pm 0.7, \mathrm{P}<0.01)$. However, other MRI and PSG assessments including retroglossal space, soft palatal length, soft palatal thickness, tongue length, high retropharyngeal area, low retro-pharyngeal area, minimum retropharyngeal area, and lateral pharyngeal wall thickness were not significantly different between patients with and without OSA. Comparison of the MRI and PSG assessments of patients with and without OSA was presented in Table 2.

Table 2. Comparison of pharyngeal measurements of patients with and without OSA.

\begin{tabular}{llc}
\hline Pharyngeal measurements & $\begin{array}{l}\text { Patients without OSA mean } \\
(\text { SD })\end{array}$ & Patients with OSA mean (SD) \\
\hline Retropalatal distance $(\mathrm{mm})$ & $5.7(1.4)$ & $4.3(0.7)$ \\
\hline Retroglossal space $(\mathrm{mm})$ & $22.9(3.1)$ & $20.3(3.1)$ \\
\hline Soft palatal length $(\mathrm{mm})$ & $36.4(4.0)$ & $39.3(3.0)$ \\
\hline Soft palatal thickness $(\mathrm{mm})$ & $11.2(2.1)$ & $12.3(1.1)$ \\
\hline Tongue length $(\mathrm{mm})$ & $66.3(6.8)$ & $70.7(3.6)$ \\
\hline High retro-pharyngeal area $\left(\mathrm{mm}^{2}\right)$ & $151.2(49.9)$ & $116.4(36.9)$ \\
\hline
\end{tabular}


Citation: Zhang L, Wu Q, Zhang Q, et al. Analysis of pharynx MRI in stroke patients with obstructive sleep apnea. Allied J Med Res. 2018;2(1):1-7.

\begin{tabular}{llll}
\hline Low retro-pharyngeal area $\left(\mathrm{mm}^{2}\right)$ & $140.3(63.8)$ & $204.2(110.7)$ & 0.13 \\
\hline Minimum retro-pharyngeal area $\left(\mathrm{mm}^{2}\right)$ & $103.9(66.4)$ & $72.9(39.6)$ & 0.17 \\
\hline Lateral pharyngeal wall thickness $(\mathrm{mm})$ & $36.9(5.4)$ & $38.8(1.6)$ & 0.25 \\
\hline
\end{tabular}

Note: SD: Standard Deviation

The Pearson's correlation coefficient (r) between each pair of pharyngeal MRI and polysomnography measurements was presented in Table 3. A greater correlation coefficient ( $r>0.5)$ was considered as substantial correlation in this study. Shorter retropalatal distance was substantially associated with higher
AHI and OAI but lower $\mathrm{LSaO} 2$, shorter retroglossal space was substantially associated with the higher CAI, and shorter tongue length was substantially associated with higher $\mathrm{LSaO} 2$. These correlations were presented in Figure 2.
$\mathbf{A}$
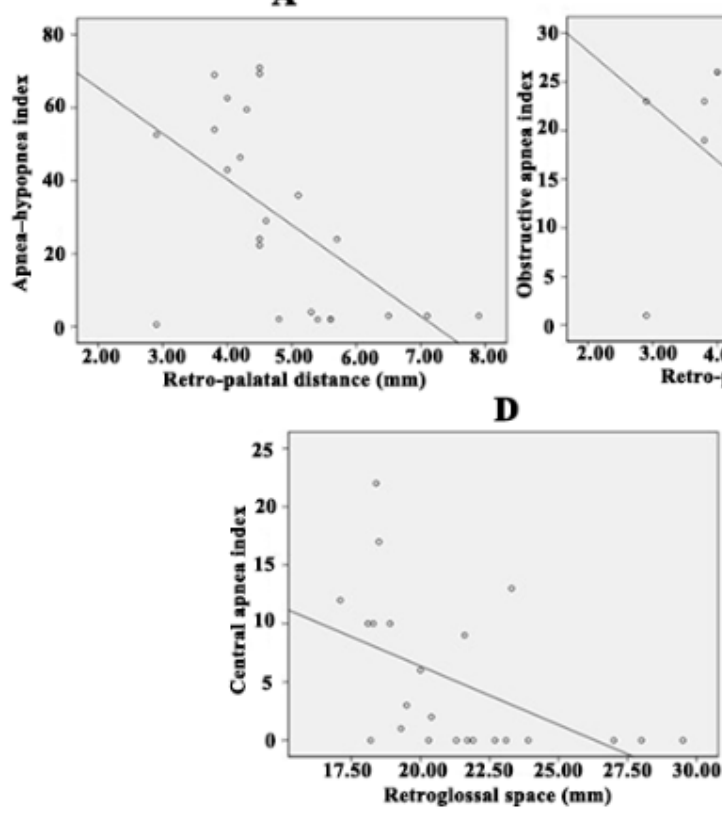

B

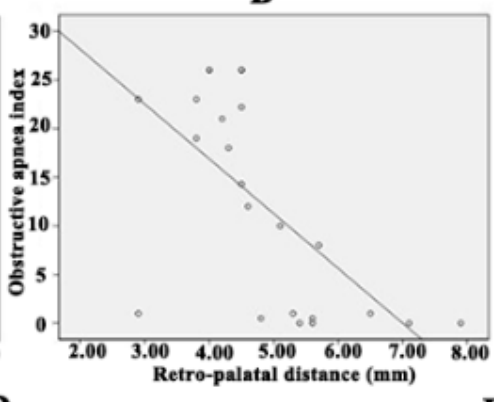

$\mathbf{E}$

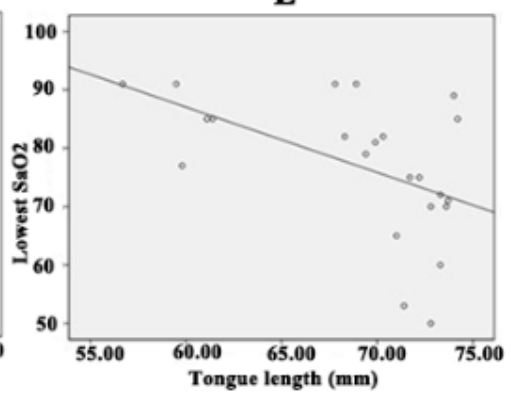

Figure 2: Scatter plot: (A) apnea-hypopnea index (Y-axis) and retro-palatal distance (X -axis); (B) obstructive apnea index (Y-axis) and retropalatal distance (X -axis); (C) Lowest $\mathrm{SaO} 2$ (Y-axis) and retro-palatal distance (X -axis); (D) central apnea index (Y-axis) and retroglossal space (X -axis); (E) Lowest $\mathrm{SaO} 2$ (Y-axis) and tongue length (X -axis).

Table 3. Pearson correlation coefficient between pharyngeal MRI and polysomnography measurements.

\begin{tabular}{|c|c|c|c|c|c|c|c|c|}
\hline \multirow{2}{*}{$\begin{array}{l}\text { Polysomnography } \\
\text { measurement }\end{array}$} & \multicolumn{8}{|c|}{ Pharyngeal MRI measurement } \\
\hline & $\begin{array}{l}\text { Retro-palatal } \\
\text { distance }(\mathrm{mm})\end{array}$ & $\begin{array}{l}\text { Retroglossal space } \\
(\mathrm{mm})\end{array}$ & $\begin{array}{l}\text { Length of soft } \\
\text { palatal }(\mathrm{mm})\end{array}$ & $\begin{array}{l}\text { Soft palatal } \\
\text { thickness }(\mathrm{mm})\end{array}$ & $\begin{array}{l}\text { Tongue } \\
(\mathrm{mm})\end{array}$ & length & $\begin{array}{l}\text { Minimum retro- } \\
\text { pharyngeal area }(\mathrm{mm} 2)\end{array}$ & $\begin{array}{l}\text { Lateral pharyngeal } \\
\text { wall thickness }(\mathrm{mm})\end{array}$ \\
\hline $\mathrm{AHI}$ & $-0.57^{\star \star}$ & $-0.46^{*}$ & $0.43^{*}$ & 0.27 & 0.41 & & -0.25 & 0.31 \\
\hline OAI & $-0.63^{* *}$ & -0.37 & $0.43^{*}$ & 0.34 & 0.41 & & -0.29 & 0.33 \\
\hline CAI & $-0.49^{*}$ & $-0.51^{*}$ & 0.2 & 0.1 & 0.29 & & -0.23 & 0.23 \\
\hline MAI & -0.32 & -0.17 & 0.28 & 0.05 & 0.36 & & -0.1 & 0.16 \\
\hline $\mathrm{HI}$ & -0.4 & $-0.46^{*}$ & 0.4 & 0.29 & 0.3 & & -0.17 & 0.27 \\
\hline ODI & -0.3 & $-0.42^{*}$ & 0.04 & 0.02 & 0.31 & & -0.29 & 0.17 \\
\hline $\mathrm{M}-\mathrm{SaO}_{2}$ & 0.3 & $0.44^{*}$ & -0.38 & -0.16 & $-0.43^{*}$ & & 0.29 & $-0.42^{*}$ \\
\hline $\mathrm{L}-\mathrm{SaO}_{2}$ & $0.59^{* *}$ & 0.29 & $-0.49^{*}$ & -0.39 & $-0.51^{*}$ & & 0.12 & -0.12 \\
\hline
\end{tabular}

Note:- AHI: Apnea-Hypopnea Index; OAI: Obstructive Apnea Index; CAI: Central Apnea Index; MAI: Mixed Apnea index; ODI: Oxygen Desaturation Index; M-Sa0 ${ }_{2}$ : Mean $\mathrm{SaO}_{2} ; \mathrm{L}-\mathrm{SaO}_{2}$ : Lowest $\mathrm{SaO}_{2}$ 


\section{Discussion}

Oropharyngeal structural abnormality is an important physiological characteristics and risk factor leading to a nonstroke OSA. In the present study, we showed that the retropalatal distance among stroke patients was longer for patients with OSA compared to those without OSA, and it is negatively related to the $\mathrm{AHI}$ and $\mathrm{OAI}$ and positively related to the $\mathrm{LSaO}_{2}$. We also showed that retroglossal space was negatively related to the $\mathrm{CAI}$ and tongue length was positively related to the $\mathrm{L}-\mathrm{SaO}_{2}$.

Why do the pharyngeal structure change in patients with OSA? Although the underlying pathogenesis is unknown, the most likely reasons are genetic factors, obesity, neuromechanical factors and the disease itself. Several studies in the current literature investigated the changes in pharyngeal anatomy among patients with non-stroke OSA. Trudo et al. [22] examined the retro-palatal distance and the retroglossal space among healthy people when they were awake and asleep. They found that when the subjects were in deep slept, their retropalatal distance became shorter substantially, while no obvious changes were observed on the retroglossal space. They believed the decreased retro-palatal distance could lead to a narrow upper airway during sleep. De Mello et al. [23] found that soft palate played a major role in airway narrowing during sleep and they believed that the decrease of upper airway muscle tone was mainly due to a shorten retro-palatal distance. In the study by Mattaias et al. [24], patients with neuromuscular dysfunction at the throat were assessed using MRI on pharynx and the surface electromyography on the upper airway dilator. They found that the retropalatal distance was shorter and the retroglossal space was smaller for patients with OSA compared to healthy people. They thought the decreasing of upper airway muscle tension was a pathophysiological mechanism of OSA. These studies suggested that the main cause of OSA was the anatomical or functional changes of the pharyngeal soft tissue, which led to a shorter retro-palatal distance and a smaller retroglossal space, and consequently resulted in the narrowing and collapse of airway during inspiration.

Some other studies showed that OSA was associated with a shorter retro-palatal distance among patients with stroke. Brown et al. [16] found that retro-palatal distance was shorter among stroke patients who had OSA compared to stroke patients who had no OSA. They also found that the shorter retro-palatal distance was associated with higher AHI and none of the other morphological characteristics differed between those with and without OSA. These are consistent with our findings. Moreover, we also found that retro-palatal distance was linearly associated with the AHI, OAI and L-SaO2, retroglossal space was linearly associated with $\mathrm{CAI}$, and tongue length was linearly associated with $\mathrm{L}-\mathrm{SaO} 2$.

The pharyngeal's anatomical morphology researches on the patients with and without stroke showed that the oropharyngeal structural abnormality was associated with the OSA, especially the retro-palatal distance. In our research we found the Retropalatal distance, Retroglossal space and Tongue length were related with the OSA $(r>0.5)$, some researchers believed the soft palate and tongue were related to a decrease in upper airway dilator muscle activity $[25,26]$, and the integrity of the retro-palatal area seems to be particularly susceptible to a decrease in upper airway dilator tone. Firstly, the retro-palatal distance of upper airway is shorter than the retroglossal area, and during inspiration the air velocity of the retro-palatal area is much higher. When palate-pharyngeal muscle paralysis, the resistance will increase more rapidly in the high-resistance retro-palatal airway area, and the higher velocity in the highresistance retro-palatal area will be accompanied by a lower local intraluminal pressure in the retroglossal space [27]. Thus, physiologic differences in the flow resistance between the retro-palatal and retroglossal area that should be particularly marked with decreased upper airway dilator tone may contribute to differences in airway volume and collapsibility. Besides, compared with the palatini (retopalatal area), the genioglossus muscle (retroglossal area) is not the predominantly tonically active tensor, which increases its activity with negative pharyngeal pressure by the negative pressure reflex [28]. Other studies showed that the most common reasons of the increasing incidence of OSA among stroke patients were the oropharyngeal muscles dysfunction and the decreasing neuromuscular control after stroke [29-32]. This may be explained by an impairment of the balance between upper airway dilating forces and negative intraluminal pressure generated during inspiration by respiratory "pump" muscles [33]. Also, some studies found that the SDB (sleep disordered breathing) was also associated with dysphagia and its Severity, which was not shown in the present study [34]. In the process of clinical diagnosis and treatment, we found that many stroke patients were accompanied OSA, and some of them also had dysphagia. This research is the first stage of our study, the further study will focus on the relationship of dysphagia, OSA and stroke.

To the best of our knowledge, brain MRI studies are part of routine clinical care and are not dedicated to studying the upper airway. MRI measurements were carried out in a standard supine position which has been found that this position itself may lead to OSA. Brain MRI scans are commonly used in the assessment of stroke patients. We were unable to measure the lateral pharyngeal soft tissue thickness on the axial sections and therefore used the coronal images to assess these parameters. However, this did not cause any effect to the results of this study since both the PSG monitoring measurements and the pharynx MRI measurements were performed with the patient supine [35], but they did not explicitly mention whether it was associated with OSA. Some OSA studies are not strongly associated with the location or severity of cerebral infarction [36,37]. Additionally, the present study did not investigate whether the onset of OSA was before or after the stroke. Future studies were needed to explore this mechanism.

Furthermore, a large body of evidence suggests that OSA has a negative impact on the immediate and long-term functional outcome after stroke. The apnea-hypopnea index (AHI), a common measure of sleep apnea severity, is an independent predictor of mortality in stroke patients $[15,16]$. Based on these findings, these important associations between OSA and 
Citation: Zhang L, Wu Q, Zhang Q, et al. Analysis of pharynx MRI in stroke patients with obstructive sleep apnea. Allied J Med Res. 2018;2(1):1-7.

stroke, and stroke outcomes underscore the need to understand the role that OSA treatment may play in stroke.

In conclusion, we found that OSA after stroke was associated with the shorter retropalatal distance. None of the other morphological characteristics were found to be significantly associated with OSA after stroke. This small change about the shorter retropalatal distance in the MRI protocols can improve the diagnostic criteria for OSA and may further explain the mechanism by which OSA is so common in stroke patients.

\section{References}

1. Young T, Palta M, Dempsey J, et al. The occurrence of sleep-disordered breathing among middle-aged adults. N Engl J Med. 1993;328:1230-35.

2. Evans JRC, McRae L, Driver H, et al. The prevalence rate and risk of obstructive sleep apnea in Canada. Chest. 2010;138:702.

3. Hermann DM, Bassetti CL. Sleep-related breathing and sleep-wake disturbances in ischemic stroke. Neurology. 2009;73:1313-22.

4. Punjabi NM. The epidemiology of adult obstructive sleep apnea. Proc Am Thorac Soc. 2008;5:136-43.

5. Addison-Brown KJ, Letter AJ, Yaggi K, et al. Age differences in the association of obstructive sleep apnea risk with cognition and quality of life. J Sleep Res. 2014;23:69-76.

6. Kendzerska T, Mollayeva T, Gershon AS, et al. Untreated obstructive sleep apnea and the risk for serious long-term adverse outcomes: a systematic review. Sleep Med Rev. 2014;18:49-59.

7. Iranzo A, Santamaria J, Berenguer J, et al. Prevalence and clinical importance of sleep apnea in the first night after cerebral infarction. Neurology. 2002;58:911-6.

8. Good DC, Henkle JQ, Gelber D, et al. Sleep-disordered breathing and poor functional outcome after stroke. Stroke. 1996;27:252-259.

9. Askenasy JJ, Goldhammer I. Sleep apnea as a feature of bulbar stroke. Stroke. 1988;19:637-9.

10. Brown DL, Lisabeth LD, Zupancic MJ, et al. High prevalence of supine sleep in ischemic stroke patients. Stroke. 2008;39:2511-4.

11. Ciscar MA, Juan G, Martínez V, et al. Magnetic resonance imaging of the pharynx in OSA patients and healthy subjects. Eur Respir J. 2001;17:79-86.

12. Rodenstein DO, Dooms G, Thomas Y, et al. Pharyngeal shape and dimensions in healthy subjects, snorers, and patients with obstructive sleep apnoea. Thorax. 1990;45:722-7.

13. Schwab RJ, Pasirstein M, Pierson R, et al. Identification of upper airway anatomic risk factors for obstructive sleep apnea with volumetric magnetic resonance imaging. Am J Respir Crit Care Med. 2003;168:522-30.

14. Eikermann M, Vogt FM, Herbstreit F, et al. The predisposition to inspiratory upper airway collapse during partial neuromuscular blockade. Am J Respir Crit Care Med. 2007;175:9-15.
15. Shigeta Y, Enciso R, Ogawa T, et al. Correlation between retroglossal airway size and body mass index in OSA and non-OSA patients using cone beam CT imaging. Sleep Breath. 2008; 12: 347-52.

16. Brown DL, Bapuraj JR, Mukherji SK, et al. MRI of the pharynx in ischemic stroke patients with and without obstructive sleep apnea. Sleep Med. 2010;11:540-4.

17. Brott T, Adams HP Jr, Olinger CP, et al. Measurements of acute cerebral infarction: a clinical examination scale. Stroke. 1989;20:864-70.

18. Trapl M, Enderle P, Nowotny M, et al. Dysphagia bedside screening for acute-stroke patients: the Gugging Swallowing Screen. Stroke. 2007;38:2948-52.

19. Johns MW. A new method for measuring daytime sleepiness: the Epworth sleepiness scale. Sleep. 1991;14:540-5.

20. Daniel MM, Lorenzi MC, da Costa LC, et al. Pharyngeal dimensions in healthy men and women. Clinics. 2007;62:5-10.

21. Rodenstein DO, Dooms G, Thomas Y, et al. Pharyngeal shape and dimensions in healthy subjects, snorers, and patients with obstructive sleep apnoea. Thorax. 1990;45:722-7.

22. Trudo FJ, Gefter WB, Welch KC, et al. State-related changes in upper airway caliber and surrounding softtissue structures in normal subjects. Am J Respir Crit Care Med. 1998;158:1259-70.

23. Mello CF, Filho HAG, Gomes CA, et al. Radiological findings in patients with obstructive sleep apnea. J Bras Pneumol. 2013;39:98-101.

24. Eikermann M, Vogt FM, Herbstreit F, et al. The predisposition to inspiratory upper airway collapse during partial neuromuscular blockade. Am J Respir Crit Care Med. 2007;175:9-15.

25. Abbott MB, Donnelly LF, Dardzinski BJ, et al. Obstructive sleep apnea: MR imaging volume segmentation analysis. Radiology. 2004;232:889-95.

26. Fogel RB, Trinder J, White DP, et al. The effect of sleep onset on upper airway muscle activity in patients with sleep apnoea versus controls. J Physiol. 2005;564:549-62.

27. White DP. Pathogenesis of obstructive and central sleep apnea. Am J Respir Crit Care Med. 2005;172:1363-70.

28. Fung YC. Biomechanics: motion, stress and growth. New York: Springer Verlag. 1990; Pp. 83-7.

29. Malhotra A, Pillar G, Fogel RB, et al. Genioglossal but not palatal muscle activity relates closely to pharyngeal pressure. Am J Respir Crit Care Med. 2000;162:1058-62.

30. Steier J, Seymour J, Rafferty GF, et al. Continuous transcutaneous submental electrical stimulation in obstructive sleep apnea: a feasibility study. Chest. 2011;140:998-1007.

31. McSharry D, O'Connor C, McNicholas T, et al. Genioglossus fatigue in obstructive sleep apnea. Respir Physiol Neurobiol. 2012;183:59-66.

32. Svatikova A, Chervin RD, Wing JJ, et al. Positional therapy in ischemic stroke patients with obstructive sleep apnea. Sleep Med. 2011;12:262-6. 
33. Martínez-García MA, Galiano-Blancart R, Soler-Cataluña $\mathrm{JJ}$, et al. Improvement in nocturnal disordered breathing after first-ever ischemic stroke: role of dysphagia. Chest. 2006;129:238-45.

34. Eikermann M, Vogt FM, Herbstreit F, et al. The Predisposition to Inspiratory Upper Airway Collapse during Partial Neuromuscular Blockade. Am J Respir Crit Care Med. 2007;175:9-15.

35. Bassetti C, Aldrich MS. Sleep apnea in acute cerebrovascular diseases: final report on 128 patients. Sleep. 1999;22:217-23.

36. Parra O, Arboix A, Bechich S, et al. Time course of sleeprelated breathing disorders in first-ever stroke or transient ischemic attack. Am J Respir Crit Care Med. 2000;161:375-80.

\section{${ }^{*}$ Correspondence to:}

Tong Wang,

Vice director, Department of Rehabilitation,

Jiangsu Provincial People's Hospital,

The First Affiliated Hospital of Nanjing Medical University

China

Tel: 17326130952

E-mail: wangtong201709@yeah.net 